BNL-45516

(Rev. 6/91)

AD/RHIC-91

Tech Note

\title{
First Turn Around Strategy for RHIC
}

\author{
J. Milutinovic and A. G. Ruggiero
}

June 1991

\section{R H I P R O E C T}

\author{
Brookhaven National Laboratory \\ Associated Universities, Inc. \\ Upton, NY 11973
}

Under Contract No. DE-AC02-76CH00016 with the UNITED STATES DEPARTMENT OF ENERGY 


\section{First Turn Around Strategy for RHIC}

\section{J. Milutinovic \& A. G. Ruggiero}

We present a strategy for achieving the so-called first turn around in RHIC. The strategy is based on the same method we had proposed to correct a distorted closed orbit in RHIC, i.e. on a generalization of the local three-bump method. ${ }^{1}$ We found out that the method is very effective in passing the beam through a non-ideal, insufficiently known, machine. We tested the software on ten different Gaussian distributions of dominant orbit distorting lattice imperfections, i.e. error in the dipole integrated strength $\Delta(B \ell) / B \ell$, axial roll of the dipole and lateral displacements of the quadrupole in each plane. The errors were introduced with realistic RMS values and a $2.5 \sigma$ cut. The perturbed lattice was generated by the code PATRIS, which was also adapted to control the newly developed software. In all of the ten distributions the software was capable of passing the beam through in 2-3 injection attempts, at full sextupole strength. It was also determined that once the beam makes the first turn around and all the correctors are energized, it stays in the machine for at least several hundred turns that we had checked. The quality of the orbit, that was established in this way, was also found to be very good, i.e. the residual distortions at the places of large beta function were much less than one millimeter. With one or two monitors/correctors broken, the software established a first turn around without any extra difficulties. The quality of such orbit was, of course, somewhat degraded, especially around the broken devices. It was also observed that, in the process of actual closing, the beam develops free betatron oscillations in the amplitude range of $1-5 \mathrm{~mm}$, which can be reduced either by changing the injection conditions to better match the actual closed orbit or by an appropriate damping device. The hardware proposed for RHIC is more than sufficient to meet the demands of the first turn controlling software. The maximum kick angle to be applied to the beam would require less than $2 / 3$ of the corrector's top strength $(0.3 \mathrm{~T} \cdot \mathrm{m})$ even at the top magnetic rigidity $B \rho=850 \mathrm{~T} \cdot \mathrm{m}$, which means that the correctors will be performing an easy task at injection.

\section{Introduction}

An accelerator lattice cannot be expected to be perfect and as an immediate consequence the same is true for the closed orbit in the machine. Since more or less reliable assumptions can be made about the order of realistic lattice imperfections, it is also possible to estimate the order of the resulting closed orbit distortions. If there is a well-defined

1 J. Milutinovic and A.G. Ruggiero, AD/RHIC/AP-78 (BNL Internal Report, 1989). 
correcting scheme, a distorted closed orbit can also be corrected to a level of distortions which are acceptable. Many correcting schemes have been proposed. Some well-known examples are harmonic correction, Micado scheme and the local three-bump method used at Fermilab. Another example is a generalization of the local three-bump method, which we propose to correct the closed orbit in RHIC, whose essentials will be presented in this technical note. All these correcting schemes have one common feature. They evaluate corrector strengths on the basis of some knowledge about the optical properties of the ideal lattice and on the basis of actual orbit readings on a set of beam position monitors installed in the machine.

This in turn requires a beam circulating in the machine, so that the orbit readings can be taken. An important consequence is the existence of a very special moment in the history of every accelerator, i.e. the situation when the machine is completed and ready to work, but the beam has yet to be injected for the first time. The problem is the unknown machine which will obviously give rise to a distorted closed orbit, but which cannot be corrected before the readings are taken. Since correctors cannot be adequately set, the beam is first injected without any correction and can easily encounter physical aperture limitations and be lost before making the first turn around. This necessitates implementation of a special strategy, called "the first turn around strategy", to complete one turn, after which one hopes to know enough about the effects of lattice imperfections to be able at least to keep the beam in the machine until the orbit can be better corrected. However, in the case of RHIC, realistic closed orbit analysis ${ }^{1,2}$ indicates that there are very good chances that the beam hits the walls of the vacuum chamber before making a full turn. Hence, developing a "first turn around strategy" is a necessity for RHIC.

\section{RHIC Lattice - Its Imperfections and Consequences on the Closed Orbit}

There are four types of lattice imperfections which are considered to be major sources of orbit distortions. They are the error in the integrated dipole field strength $\Delta(B \ell) / B \ell$, the axial rotation of the dipole $\Delta \theta$, and the lateral displacements $\Delta_{Q} X, \Delta_{Q} Y$ of the quadrupole in the two transverse directions.

The RMS values of the lattice errors applicable to RHIC are the following ones:

$$
\begin{gathered}
\Delta(B \ell) / B \ell=0.5 \times 10^{-3}, \quad \Delta \theta=10^{-3} \mathrm{rad} \\
\Delta_{Q} X=\Delta_{Q} Y=0.25 \times 10^{-3} \mathrm{~m} .
\end{gathered}
$$

We have simulated the closed orbit distortions in RHIC, with the above RMS values and a $2.5 \sigma$ cut in Gaussian distributions of random errors in the lattice. We used PATRIS

2 A.G. Ruggiero, AD/RHIC/AP-90 (BNL Internal Report, 1990). 
as the code of choice. Throughout the simulation sextupoles were assumed to be thin lenses, but otherwise perfect, higher order nonlinearities were absent, and the effects of errors were realistically incorporated into the $7 \times 7$ transfer matrix used by PATRIS. Beam position monitors were assumed ideal, i.e. perfectly aligned with the axis going through an ideally placed quadrupole and having a perfect sensitivity. They were placed beside each quadrupole and measured orbit distortions in the plane where beta function was large. Correctors were modeled as thin lenses, but otherwise they were also considered ideal, i.e. ideally placed like BPM's and having a perfect adjustability. They were also placed beside each quadrupole, to act in the plane where beta function is large, but not at the same side of the quadrupole as BPM's. It must be emphasized that this separation of the positions of the members of a pair BPM - corrector, by a quadrupole and in the arcs also a sextupole placed inbetween, involved additional complications which we eliminated by generalizing the local three-bump method of orbit correction.

The results of our realistic closed orbit analysis showed the following characteristic features, tested on 10 different Gaussian distributions of lattice errors. With the accepted RMS values, with no correction and with no checks for possible violations of physical aperture restrictions, largest orbit distortions reached $\sim 40-50 \mathrm{~mm}$ at some BPM's in the arcs and $\sim 100 \mathrm{~mm}$ in the insertions. This means that the beam would have good chances to violate aperture limitations at some point and strike the wall of the vacuum chamber. This was one of the characteristic properties of the uncorrected closed orbit, but the same would happen during injection, resulting therefore in a beam loss and in a failure to make the very first turn around in the absence of any corrections.

\section{Methods of Achieving the First Turn Around}

There are many ways of getting the beam around for the first time. The basic task is to give the correctors adequate strengths to prevent the beam from exceeding the various aperture limitations in the machine. Demands are less stringent than those appearing in the case of correcting an already established closed orbit, since one is here primarily interested in making the very first turn around, rather than being concerned about the quality of the orbit. On the other hand, with an established closed orbit one knows more about the machine, and precisely the absence of this knowledge appears as one of the principal obstacles which must be overcome in steering the beam for the first time through the new machine.

Even though it is not necessary, one is tempted to use some of the methods developed for the purpose of closed orbit correction and adapt them to make the first turn around. However, some restrictions do apply here. Closed orbit correction methods that require 
circulating beam are obviously inappropriate, while others may be applicable with more or less success. It is worthwhile to mention that the local three-bump method ${ }^{1}$ is one of the best methods and is easily adapted to steer the beam through the machine for the first time. Indeed, this was the method of our choice and we initially developed software based on this method. It worked very well. With the RHIC's realistic errors, and with the sextupoles at full strength, the software accomplished the first turn around in just 2 or 3 injection attempts and subsequently closed and further corrected the orbit to distortions being less than one millimeter at BPM's.

There is, however, one big obstacle to implementing this conceptually elegant approach in RHIC. Based on some specific considerations, RHIC has a somewhat peculiar arrangement of its BPM's and orbit correctors. As we have already mentioned, they are being placed at the two opposite ends of each quad and not beside each other, as is the case with most other machines. For a machine with quadrupole strengths and anticipated RMS error values like RHIC, this is enough to invalidate the simple and elegant three-bump method, as our more recent studies of realistic closed orbit in RHIC clearly demonstrated. The local three-bump method requires that the beam be kicked precisely at the places where its distortions are being observed, and a quadrupole placed inside a monitor-corrector pair upsets this too much. As a result of this, the beam is kicked at a "wrong" place and is therefore not corrected properly.

The foregoing situation necessitated either a modification of the previously very successful correcting scheme, to accommodate the RHIC hardware, or modification of the hardware itself. To avoid all potential problems with hardware redesign, we attempted and succeeded in generalizing the local three-bump method to a somewhat nonlocal but nevertheless equally efficient method of closed orbit correction. The same method then we also applied to our "first turn around strategy" for RHIC, again with the same success as before. Here we summarize its essentials.

Our starting point is the formula (4.7) for closed orbit distortions, in the absence of nonlinearities, given in the Courant-Snyder paper. ${ }^{3}$ If the errors can be reasonably represented as delta functions or kicks it goes over into ${ }^{2}$

$$
Z_{i}=\frac{\sqrt{\beta_{i}}}{2 \sin \pi \nu} \sum_{j=1}^{n} \sqrt{\beta_{j}} \cos \nu\left(\varphi_{i}-\varphi_{j}-\pi\right) \theta_{j}
$$

with the supplementary condition that phase advances are ordered so that $\varphi_{i}>\varphi_{j}$ is always fulfilled. This describes the effects of kicks at $j$-th locations $(j=1,2 \ldots n)$ on the orbit distortion at i-th location $Z_{i}$. In this formula $\nu$ is the tune in the plane described

${ }^{3}$ E.D. Courant and H.S. Snyder, Anals. of Physics, 3, 1-48 (1958). 
by the above expression, $\theta_{j}$ is the effective kick at the $\mathrm{j}$-th location, expressed through the resulting kick angle, $\beta$ is the appropriate beta function and $\varphi$ the phase advance. Subscript $j$ refers to the perturber, while $i$ refers to the point of observation. In this formula, the effects of the perturbations, expressed through $\theta_{j}$, are linearly propagated along the lattice, whose linear characteristics are in this formula still considered ideal. Of course, these $\theta_{j}$ need not be the actual lattice errors. They can also be deliberately delivered kicks, which will still produce orbit distortions in an otherwise ideal lattice.

Now consider a non-ideal lattice with many errors which produce orbit distortions $R_{i}$, read at $n$ BPM's. To maximize the effectiveness of BPM's we place them beside each quadrupole where the relevant beta function is large. The actual errors will produce effects mainly described by an expression of type (2) and partially described by various nonlinear contributions. Obviously, we do not know the exact nature, position and the magnitude of each error in the actual lattice. However, we do have the readings $R_{i}$ and we can try to deliberately kick the beam so as to steer it through reflected distortions $-R_{i}$, in the absence of actual errors. When applied, these kicks will then act toward cancelling the effects of actual errors to the leading order.

Although various approaches are possible, the simplest one in both conceptual and operational sense is to introduce one corrector for each monitor in the lattice and to place one of these correctors beside each quadrupole where the relevant beta function is large. Two possibilities will arise here. Either we place a corrector at the same side of each quadrupole as the corresponding BPM, in which case we are back to the simple threebump method, as Table 1 along with its comments indicates, or we place it at the other end of each quad. In this case we accommodate the RHIC design.

Now we simply take $R_{1}, R_{2}, \ldots R_{n}$, the readings at $n$ BPM's, and demand that the $n$ corresponding correctors deliver such kicks, expressed as angles $\theta_{1}, \theta_{2}, \ldots \theta_{n}$, that they generate orbit distortions equal in magnitude but opposite in sign to those being measured, i. e. $Z_{i}=-R_{i}$. The expression (2) now yields:

$$
Z_{i}=-R_{i}=\frac{\sqrt{\beta_{i}}}{2 \sin \pi \nu} \sum_{j=1}^{n} \sqrt{\beta_{j}} \cos \nu\left(\varphi_{i}-\varphi_{j}-\pi\right) \theta_{j}=\sum_{j=1}^{n} A_{i j} \theta_{j}
$$

Since the number of correctors is the same as the number of monitors, the matrix $A$ is a square, generally nonsingular matrix which can be inverted. After inverting it, we get the desired kick angles

$$
\theta_{i}=\sum_{j=1}^{n}\left(A^{-1}\right)_{i j} Z_{j}=\sum_{j=1}^{n}\left(A^{-1}\right)_{i j}\left(-R_{j}\right)
$$


which would steer the beam through the positions $Z_{i}=-R_{i}, i=1,2, \ldots n$, in the absence of errors and will consequently cancel distortions to the leading order in the presence of errors along with nonlinearities. Without nonlinearities the cancellation is complete.

The only awkward element in this scheme is inversion of a big $123 \times 123$ matrix $A$ for RHIC and some delocalization of the dependence of $\theta_{i}$ on the BPM readings in its vicinity. However, matrix inversion has proved to be no problem on modern computers and the delocalization we mentioned is not drastic, either. Therefore, both problems have fortunately turned out to be very minor for RHIC, and that resulted in a very successful handling of the realistic closed orbit problem. We would also mention that once the matrix has been inverted the new scheme's performance equals the performance of the simpler three-bump method in the presence of sextupoles and, just as the previous scheme, it reduces orbit distortions to zero at all BPM's in the absence of nonlinearities. We would also mention in passing that from the programming point of view the new scheme enjoys the advantage of allowing somewhat simpler and more elegant software, whose adaptation to achieve the first turn around is straightforward.

\section{Implementation of the Method}

Now the strategy goes as follows. The beam is injected and its progressing through the lattice is monitored on BPM's. The correctors are turned off, since initially one does not have any information on how to power them. Once the beam is lost by exceeding the available aperture at a certain place in the lattice, whose exact location is not essential for the strategy, one knows the readings on all BPM's preceding the area where the beam is lost. Only these readings are introduced into the expression (4) and not any other kind of information, except that the orbit coordinates at the BPM's past the point of loss, where no readings have appeared yet, are simply left to be zero. The expression (4) then predicts fairly accurate values of kick angles for all correctors except for a couple of them around the injection point and a couple of them around the point where the beam is lost. But even in these two regions the predicted values of kick angles are not in a violent disagreement with the actual values, except for the very first and the very last corrector which are then not energized at this stage. All the other correctors between the injection point and the point where the beam is lost are then energized and the beam is injected again. It then starts with significantly reduced distortions, passes the critical point, and continues along the lattice in the region with the correctors still not energized until it is lost again. The whole procedure of kick angle evaluation is now repeated and the newly found kick strengths are simply added to the previous values. The correctors are then energized again, with more of them being powered at this stage, and the beam is reinjected. This procedure is then 
continued until the beam makes its first turn around. With RHIC's RMS error levels, under ideal injection conditions assumed so far in the simulations, it usually takes just 2 or 3 injection attempts to make the first turn around. The results of the whole procedure for a typical case are displayed in Tables $3,4,5$.

Once the beam makes the first turn around, it usually fails in making the second turn, since the first turn is made with part of the correctors not energized yet. But on the basis of the first turn being achieved, one has all the necessary information to adequately energize all of the correctors, except several in the vicinity of the injection point. However, even here only the first and the last one have their predicted strengths in a complete disagreement with the proper values. These proper values were found previously in an independent simulation of closed orbit distortions on the same lattice, a procedure which cannot be mimicked on the real machine before a closed orbit has been even established! So at this stage one energizes all of the correctors and allows the beam to circulate. Simulations indicate that then the beam survives for at least several hundred turns that were checked and it can therefore be concluded that it has displayed at least a short-term stability within the available apertures.

There is another problem that shows up now. The orbit is established and its distortions are significantly reduced, but it is not completely corrected yet. That means that the injection point does not lie at the current, mostly corrected closed orbit. As a result, betatron oscillations develop the very moment the beam makes the first turn around. This is shown in Tables 6 and 7. To prove that the large readings at the BPM's, which change from turn to turn, are really betatron oscillations, we plotted the readings at a fixed BPM for several dozen turns and observed that they lie on a typical phase space ellipse (Fig. 1). That not only proved that we are indeed dealing with betatron motion but also supported our conclusion that the beam maintains at least a short-term stability once all of the correctors have been energized for the first time.

The remaining problem is now the adjustment of the correctors around the injection point, which so far have not been adjusted very well. A special procedure is needed here because of betatron oscillations. They must be handled with care since they do not contribute to the values of kick angles predicted by (4), provided some continuity conditions are respected. To be specific, all of the BPM readings determining a particular kick angle $\theta_{k}$ must lie on a single continuous segment of betatron motion displacements. For the very first kick angle $\theta_{1}$, this means that $R_{1}, R_{2}, \ldots R_{n}$ of a single pass are inadequate, since the $n$ th reading $R_{n}$ and the first reading $R_{1}$ are not part of a sequence of betatron displacements running continuously across the injection point. Therefore, one has to ensure continuity of betatron displacements by taking several displacements from the end of a particular 
turn and several displacements from the beginning of the next turn. One has to take the sequence $R_{n-m+1}^{k}, R_{n-m+2}^{k}, \ldots R_{n-1}^{k}, R_{n}^{k}, R_{1}^{k+1}, R_{2}^{k+1}, \ldots R_{m}^{k+1}$, with $k$ meaning the turn's number (i.e. $k$ for $k$-th turn, $k+1$ for $(k+1)$-th one), $n$ meaning the total number of monitors and $m$ denoting the number of terms on each side of the injection point. The last number $m$ will depend on the degree of delocalization of the dependence of $\theta_{j}$ on the neighboring BPM readings. Fortunately, the problem is only mildly delocalized for RHIC, which means that in the inverse matrix $A^{-1}$ the magnitudes of its elements decrease very rapidly as we move away from its diagonal. Table 2 clearly demonstrates this property for $j=10$. For the kick angle $\theta_{j}$, it appears that the dominant matrix elements are $\left(A^{-1}\right)_{j, j-1}$, $\left(A^{-1}\right)_{j j},\left(A^{-1}\right)_{j, j+1}$, just the only nonvanishing group in the case of the simple three-bump method (Table 1), and others decrease by one order of magnitude or better as we move one more monitor away from $(j-1)$-th or $(j+1)$-th monitor. Therefore, the value of a particular kick angle $\theta_{j}$ is determined by only $4-5$ monitor readings before and $4-5$ readings after $j$-th monitor which is paired with $j$-th corrector. This is very fortunate since a totally delocalized dependence would make impossible any selection of a reduced subset (i.e. $2 \mathrm{~m}<\mathrm{n}$ ) of BPM's straddling the injection point and providing a continuous readout of betatron displacements. Any selection of monitors would have to include all monitors in the ring and this would involve a discontinuity, caused by nonclosure of the orbit with a finite betatron amplitude for any noninteger tune, and this discontinuity would give rise to spurious contributions to $\theta_{j}$ evaluated by (4). In such a case, one would have to devise a method of filtering out the betatron oscillations by determining them with a sufficient accuracy from readings over several turns and subtracting them. Alternatively, readings could be taken over very many turns and averaged for each particular BPM over these turns so that the betatron component contributions to the averaged displacements die out and only the real closed orbit distortions remain to be then used in (4). But due to a limited number of readings that contribute to each $\theta_{j}$, we simply take the readings from $m$ BPM's before the injection point and $m$ BPM's after the injection point, from the next turn, of course. This specially chosen string of BPM readings is then used in (4) to adjust those correctors which were relatively poorly adjusted upon the first orbit closing, due to the discontinuity associated with betatron motion in the original sequence $R_{1}, R_{2}, \ldots R_{n}$. In practice $m=10$ is more than enough and only 5 correctors at each side of the injection point (in each plane) require this extra treatment until they are finely tuned like the others in the lattice. Several turns which the beam makes anyway after the completion of the first turn are more than enough for the special procedure of final adjustments, and after the completion of this procedure all the correctors are nicely adjusted to the excitations that a fully corrected closed orbit would require. 
At this stage, however, sizable readings, in a 1 - $5 \mathrm{~mm}$ range, may still appear at most monitors. But our analysis demonstrates that they are essentially $100 \%$ betatron oscillations which arise because one still does not inject at the actual closed orbit with $100 \%$ accuracy. These betatron oscillations are not desirable and would have to be removed either by an appropriate damping device ${ }^{4}$ or by trying to adjust the initial injection conditions to better match the actual closed orbit.

For the purpose of simulation, we developed a simple algorithm which on the basis of linear optics properties of the lattice and on the basis of some readings at BPM's evaluates the initial betatron amplitude and phase at the injection point, and then subsequently finds the actual closed orbit at the injection point by subtracting the betatron component out. With this knowledge one can try to adjust the injection initial conditions so that they better match the currently corrected (nonideal) closed orbit. After this readjustment, the betatron motion is significantly reduced, i.e. by one order of magnitude at least. Tables 6 and 7 demonstrate betatron oscillations occurring as the beam closes. Table 8 shows the effects of readjustment of the injection conditions.

\section{Actual Performance of the Software}

We developed a program for carrying out the "first turn around strategy". We installed it in the computer code PATRIS which served as a simulator that replaced a real machine. Special modules were built in PATRIS to simulate the progress of beam going around the lattice, aperture checks and BPM readings. Another module, based on expression (4), was developed to evaluate the kick angles $\theta_{j}$ which were necessary to prevent the beam loss at certain point in the lattice. This module is capable of working on a real machine, once it knows the linear optics properties of the lattice by reading them from an appropriate database, and is completely independent of PATRIS and the ways it simulates closed orbit errors. The only item the module needs is a sequence of BPM readings.

We performed many stringent tests of the software we developed. First of all, we wanted some redundancy so that the software can do more than just a bare minimum, provided there is enough strength available from the correctors to kick the beam properly. We tested this redundancy by shrinking the apertures to just $10 \%$ of their nominal values and tried to get the beam around. The software worked fine. Of course, many more injection attempts were needed since the beam was lost much more frequently than under the realistic operating conditions, but otherwise everything was normal. It is worthwhile

4 J. Xu, et al., "The Transverse Damper System of RHIC", 1991 IEEE Particle Accelerator Conference, San Francisco, CA, May 6-9, 1991. 
to mention that these tests were being performed with the sextupoles at their full strengths and with realistic lattice errors.

To verify our conclusion that the sizable displacements that appear at most monitors after the beam completes the first turn around are almost entirely attributable to free betatron motion, we extracted the $\mathrm{X}^{\prime}$ value at one of the monitors, something impossible in a real machine but trivial in a computer simulation, along with the observable displacement $\mathrm{X}$, for several hundred subsequent turns after the first one and plotted the data. A typical phase space ellipse (Fig. 1) appears and confirms that we indeed have predominantly betatron motion. The ellipse is very slightly off-centered since it lies on the actual and not design closed orbit. This exercise also demonstrated at least a short-term stability of the beam after the injection.

Next stage was a large-scale testing. It was done for 10 different Gaussian distributions of random lattice errors, with RMS values given by (1) and with a $2.5 \sigma$ cut. The results were overwhelmingly positive. In most cases the code achieved its objective of making the first pass around in just 2 or 3 injection attempts. A little bit more was then needed for fine tuning of several correctors, only around the injection point, and for reduction of the magnitude of betatron motion. All this was done with the sextupoles at their full strengths, and these results are very encouraging since it is known that sextupoles tend to undermine the effectiveness of a linear correcting scheme. It is worthwhile to mention that the quality of the orbit, established in this way, was excellent and only minute further corrections and adjustments may be needed. The results of these injection attempts, until the first turn is established are shown in Tables 3 through 7. In Table 7, which represents the situation after the first turn, the emergence of betatron oscillations in the last column is obvious. Table 8 shows the reduction of betatron motion after a readjustment of the injection conditions.

Another important point is that the maximum kick angle that we encountered was $\theta_{\text {max }}=0.18 \mathrm{mrad}$. Even at the top magnetic rigidity of $B \rho=850 \mathrm{~T} \cdot \mathrm{m}$ this angle would require the integrated strength of the corrector to be

$$
\delta(B \ell)=(B \rho) \theta_{\max }=0.17 \mathrm{~T} \cdot \mathrm{m}
$$

which is only about $60 \%$ of the correctors' top strength of $0.3 \mathrm{~T} \cdot \mathrm{m}$, and this obviously means that at much lower injection energies the first turn around strategy that we propose here will not take the proposed hardware to its operational limits.

Finally, we would like to mention that we tested our software with one or two monitors malfunctioning in which case the corresponding correctors were not energized. The software was capable of establishing the first turn around without any difficulties. Only the 
quality of the orbit established under these conditions was somewhat degraded, primarily in the immediate vicinity of broken devices.

\section{Conclusion}

We have developed a linear method of orbit correction which is more general than the simple three-bump method. As a minor drawback, it involves a large $123 \times 123$ matrix inversion for each plane, but on the other hand offers a very effective way of both establishing the first turn around and later corrections of the orbit. The quality of the orbit, established with the sextupoles at their full strengths, is excellent - under ideal simulating conditions which include BPM's ideal in both sensitivity and placements, ideally placed and controlled correctors, simulated as thin lenses, ideal sextupoles which are also simulated as thin lenses, and no higher order nonlinearities, but with otherwise quite realistic lattice errors, known as the main cause of closed orbit distortions.

The software is modular and can be easily transferred to a control system. The matrix $A$ from expression (3) is based on ideal optical properties of the lattice, and its inversion would normally have to be done only once for each plane. If found convenient from the operational point of view, this inverted matrix might be stored in a database. In such a case, this inversion would have to be repeated only if some monitors are broken, since then the corresponding rows and columns in the original matrix $A$ would have to be deleted and the remaining matrix elements relabeled before the inversion. Otherwise the code would assume a vanishing displacement at the broken monitor and would incorrectly predict not only the kick angle at the corrector which is closest to the broken monitor but also kick angles at the neighboring correctors, predominantly at the two nearest neighbors. But this matrix inversion was not found to be prohibitively expensive. It took only $70 \mathrm{msec}$ of the CRAY-XMP CPU time or $3.6 \mathrm{sec}$ of the VAX $6450 \mathrm{CPU}$ time.

To conclude, we were pleased to notice that the method not only establishes the first turn around but also precorrects the orbit and, furthermore, that it guarantees the first turn around even with one or two monitors or correctors broken. In the last case, of course, the quality of the "precorrected orbit" is somewhat degraded. 
Table 1: Row No. 10 of the $A^{-1}$ Matrix for BPM's and Orbit Correctors at Identical Positions -Equivalent to the Local Three Bump Method. A Sharp Localization around $A_{10,10}^{-1}$ Clearly Displayed.

\begin{tabular}{|c|c|c|}
\hline$A_{10,1 \ldots 41}^{-1}$ & $A_{10,42 .}^{-1}$ & $A_{10,83 \ldots 123}^{-1}$ \\
\hline $0.2620360 \times 10^{-14}$ & $-.4626685 \times 10^{-15}$ & $0.3125336 \times 10^{-14}$ \\
\hline $0.2071569 \times 10^{-13}$ & $-.5800709 \times 10^{-16}$ & $0.1970782 \times 10^{-14}$ \\
\hline$-.6505870 \times 10^{-14}$ & $-.1649924 \times 10^{-15}$ & $0.2016802 \times 10^{-13}$ \\
\hline $0.1615995 \times 10^{-13}$ & $0.1213979 \times 10^{-15}$ & $-.1195507 \times 10^{-14}$ \\
\hline$-.3680753 \times 10^{-14}$ & $-.2981587 \times 10^{-13}$ & $0.1921108 \times 10^{-13}$ \\
\hline $0.1580126 \times 10^{-13}$ & $-.3492123 \times 10^{-14}$ & $0.6325268 \times 10^{-16}$ \\
\hline$-.2763068 \times 10^{-14}$ & $-.2462782 \times 10^{-13}$ & $0.1251256 \times 10^{-14}$ \\
\hline $0.2312277 \times 10^{-14}$ & $-.7596565 \times 10^{-15}$ & $0.6651898 \times 10^{-15}$ \\
\hline $0.1413144 \times 10^{-1}$ & $-.7857369 \times 10^{-13}$ & $-.2550621 \times 10^{-14}$ \\
\hline $0.1414430 \times 10^{-1}$ & $-.2478042 \times 10^{-13}$ & $-.1831118 \times 10^{-13}$ \\
\hline $0.1588457 \times 10^{-1}$ & $-.1380950 \times 10^{-13}$ & $0.6746980 \times 10^{-13}$ \\
\hline$-.1290828 \times 10^{-12}$ & $-.9864729 \times 10^{-14}$ & $-.6557880 \times 10^{-13}$ \\
\hline$-.3623207 \times 10^{-13}$ & $0.4345603 \times 10^{-13}$ & $0.8932258 \times 10^{-14}$ \\
\hline $0.1294004 \times 10^{-14}$ & $0.6624620 \times 10^{-15}$ & $-.5006733 \times 10^{-14}$ \\
\hline$-.1205209 \times 10^{-13}$ & $-.1391784 \times 10^{-13}$ & $0.7953358 \times 10^{-14}$ \\
\hline $0.4676343 \times 10^{-15}$ & $-.8155049 \times 10^{-14}$ & $-.5142902 \times 10^{-14}$ \\
\hline$-.1929754 \times 10^{-13}$ & $-.3989552 \times 10^{-14}$ & $0.7404918 \times 10^{-14}$ \\
\hline $0.3151447 \times 10^{-13}$ & $-.7309303 \times 10^{-14}$ & $-.2432921 \times 10^{-15}$ \\
\hline$-.2084929 \times 10^{-13}$ & $-.2293317 \times 10^{-15}$ & $0.1894401 \times 10^{-15}$ \\
\hline$-.1526448 \times 10^{-13}$ & $0.2967070 \times 10^{-13}$ & $-.1051910 \times 10^{-15}$ \\
\hline$-.1173087 \times 10^{-13}$ & $-.7838491 \times 10^{-15}$ & $-.2900611 \times 10^{-15}$ \\
\hline$-.4505474 \times 10^{-13}$ & $-.2281554 \times 10^{-14}$ & $-.5771117 \times 10^{-14}$ \\
\hline$-.1458610 \times 10^{-13}$ & $0.9408104 \times 10^{-16}$ & $-.1444810 \times 10^{-15}$ \\
\hline $0.6587396 \times 10^{-16}$ & $-.3767521 \times 10^{-13}$ & $-.5993010 \times 10^{-14}$ \\
\hline$-.1122464 \times 10^{-14}$ & $0.2040505 \times 10^{-14}$ & $0.2958294 \times 10^{-16}$ \\
\hline $0.3991323 \times 10^{-13}$ & $-.3670860 \times 10^{-13}$ & $-.2063296 \times 10^{-15}$ \\
\hline $0.7277474 \times 10^{-14}$ & $0.5339485 \times 10^{-15}$ & $0.4551658 \times 10^{-14}$ \\
\hline $0.9534706 \times 10^{-14}$ & $-.1534800 \times 10^{-15}$ & $0.6897878 \times 10^{-14}$ \\
\hline $0.2608543 \times 10^{-13}$ & $0.4868732 \times 10^{-16}$ & $0.1361096 \times 10^{-13}$ \\
\hline $0.6703764 \times 10^{-13}$ & $0.3820523 \times 10^{-14}$ & $-.5668968 \times 10^{-13}$ \\
\hline$-.1075958 \times 10^{-12}$ & $-.5011502 \times 10^{-14}$ & $0.3137930 \times 10^{-13}$ \\
\hline $0.1242656 \times 10^{-14}$ & $0.3172500 \times 10^{-15}$ & $-.5047378 \times 10^{-13}$ \\
\hline $0.6921750 \times 10^{-15}$ & $-.3503774 \times 10^{-15}$ & $-.4677473 \times 10^{-13}$ \\
\hline$-.1701643 \times 10^{-14}$ & $-.9243338 \times 10^{-15}$ & $-.2317714 \times 10^{-13}$ \\
\hline $0.5102417 \times 10^{-15}$ & $0.2856975 \times 10^{-15}$ & $-.3253520 \times 10^{-13}$ \\
\hline $0.2481681 \times 10^{-13}$ & $-.6375676 \times 10^{-15}$ & $-.1441637 \times 10^{-14}$ \\
\hline$-.1442293 \times 10^{-14}$ & $0.3234348 \times 10^{-15}$ & $-.5348694 \times 10^{-13}$ \\
\hline $0.2581060 \times 10^{-13}$ & $0.2607071 \times 10^{-15}$ & $0.7907403 \times 10^{-14}$ \\
\hline $0.2060528 \times 10^{-13}$ & $0.4268942 \times 10^{-14}$ & $-.1485492 \times 10^{-15}$ \\
\hline$-.7359906 \times 10^{-15}$ & $0.1346183 \times 10^{-14}$ & $0.1779459 \times 10^{-13}$ \\
\hline $0.2083131 \times 10^{-13}$ & $0.5118655 \times 10^{-14}$ & $0.3504657 \times 10^{-13}$ \\
\hline
\end{tabular}


Table 2: Row No. 10 of the $A^{-1}$ Matrix for BPM's and Orbit Correctors at Nonidentical Positions - Separated by a Quadrupole and a Sextupole. A Moderate Delocalization around $A_{10,10}^{-1}$ Clearly Displayed.

\begin{tabular}{|c|c|c|}
\hline$A_{10,1 \ldots 41}^{-1}$ & $\overline{A_{10,42 \ldots 82}^{-1}}$ & $\overline{A_{10,83}^{-1} .}$ \\
\hline$-.6563243 \times 10^{-14}$ & $-.1233212 \times 10^{-15}$ & $0.2309647 \times 10^{-14}$ \\
\hline$-.1404420 \times 10^{-12}$ & $-.4307115 \times 10^{-14}$ & $-.3195748 \times 10^{-13}$ \\
\hline $0.5356536 \times 10^{-11}$ & $-.3673302 \times 10^{-14}$ & $-.6890066 \times 10^{-14}$ \\
\hline$-.2341113 \times 10^{-9}$ & $-.3265098 \times 10^{-16}$ & $-.2642722 \times 10^{-13}$ \\
\hline $0.1022864 \times 10^{-7}$ & $0.2581637 \times 10^{-13}$ & $-.1155526 \times 10^{-13}$ \\
\hline$-.4469375 \times 10^{-6}$ & $0.3280491 \times 10^{-14}$ & $-.2313834 \times 10^{-13}$ \\
\hline $0.2407276 \times 10^{-4}$ & $0.2886492 \times 10^{-13}$ & $-.1760675 \times 10^{-13}$ \\
\hline$-.9626439 \times 10^{-3}$ & $-.9308682 \times 10^{-14}$ & $0.1103145 \times 10^{-13}$ \\
\hline $0.1445440 \times 10^{-1}$ & $-.2346387 \times 10^{-14}$ & $-.1461108 \times 10^{-13}$ \\
\hline $0.1346865 \times 10^{-1}$ & $-.9789746 \times 10^{-14}$ & $0.4762943 \times 10^{-13}$ \\
\hline $0.1842875 \times 10^{-1}$ & $-.1289620 \times 10^{-13}$ & $-.3740710 \times 10^{-12}$ \\
\hline$-.1621592 \times 10^{-2}$ & $0.7930776 \times 10^{-15}$ & $0.3118705 \times 10^{-12}$ \\
\hline $0.2547931 \times 10^{-4}$ & $0.1362966 \times 10^{-15}$ & $-.8030885 \times 10^{-13}$ \\
\hline$-.1182511 \times 10^{-5}$ & $0.9613616 \times 10^{-14}$ & $0.3771187 \times 10^{-14}$ \\
\hline $0.3427606 \times 10^{-7}$ & $0.1786649 \times 10^{-14}$ & $-.2061699 \times 10^{-13}$ \\
\hline$-.6258719 \times 10^{-9}$ & $0.4331626 \times 10^{-14}$ & $-.2880520 \times 10^{-14}$ \\
\hline $0.1439662 \times 10^{-10}$ & $-.9791002 \times 10^{-15}$ & $-.8851229 \times 10^{-15}$ \\
\hline$-.3617015 \times 10^{-12}$ & $0.4424420 \times 10^{-14}$ & $-.1007471 \times 10^{-14}$ \\
\hline $0.2468144 \times 10^{-13}$ & $-.6503293 \times 10^{-14}$ & $0.1972511 \times 10^{-13}$ \\
\hline $0.1568071 \times 10^{-13}$ & $0.8713998 \times 10^{-14}$ & $-.1436348 \times 10^{-13}$ \\
\hline $0.7802853 \times 10^{-14}$ & $-.1622156 \times 10^{-14}$ & $-.1976367 \times 10^{-14}$ \\
\hline $0.4515919 \times 10^{-13}$ & $-.1978762 \times 10^{-14}$ & $0.8329600 \times 10^{-14}$ \\
\hline $0.9726901 \times 10^{-14}$ & $-.3994290 \times 10^{-15}$ & $-.2163655 \times 10^{-13}$ \\
\hline $0.1532473 \times 10^{-13}$ & $-.3613772 \times 10^{-13}$ & $0.2886241 \times 10^{-13}$ \\
\hline$-.6315576 \times 10^{-15}$ & $-.3452085 \times 10^{-14}$ & $-.1762573 \times 10^{-13}$ \\
\hline$-.1185726 \times 10^{-13}$ & $-.3596261 \times 10^{-13}$ & $0.2268338 \times 10^{-13}$ \\
\hline$-.3220089 \times 10^{-14}$ & $0.7866305 \times 10^{-14}$ & $-.1621116 \times 10^{-13}$ \\
\hline$-.9568346 \times 10^{-14}$ & $-.3923749 \times 10^{-13}$ & $0.2551707 \times 10^{-15}$ \\
\hline$-.3987844 \times 10^{-13}$ & $0.3903046 \times 10^{-14}$ & $-.4094483 \times 10^{-15}$ \\
\hline $0.1070798 \times 10^{-12}$ & $0.2053442 \times 10^{-12}$ & $-.3069880 \times 10^{-13}$ \\
\hline$-.1158303 \times 10^{-12}$ & $-.2224082 \times 10^{-12}$ & $0.4541613 \times 10^{-13}$ \\
\hline $0.2115196 \times 10^{-13}$ & $0.6875835 \times 10^{-13}$ & $0.1239127 \times 10^{-13}$ \\
\hline $0.1381895 \times 10^{-13}$ & $0.7449459 \times 10^{-14}$ & $0.1023942 \times 10^{-13}$ \\
\hline $0.2289622 \times 10^{-14}$ & $0.5775994 \times 10^{-13}$ & $0.1890074 \times 10^{-13}$ \\
\hline$-.1120523 \times 10^{-14}$ & $-.2712919 \times 10^{-13}$ & $0.1393133 \times 10^{-13}$ \\
\hline $0.7365006 \times 10^{-14}$ & $-.1690561 \times 10^{-13}$ & $0.3681015 \times 10^{-14}$ \\
\hline $0.1552778 \times 10^{-13}$ & $-.3397324 \times 10^{-13}$ & $0.3303405 \times 10^{-13}$ \\
\hline$-.3390694 \times 10^{-14}$ & $-.1098005 \times 10^{-13}$ & $0.1534697 \times 10^{-13}$ \\
\hline $0.1635264 \times 10^{-13}$ & $-.2798542 \times 10^{-13}$ & $-.7700626 \times 10^{-15}$ \\
\hline$-.2410049 \times 10^{-14}$ & $0.3366792 \times 10^{-14}$ & $-.4741820 \times 10^{-15}$ \\
\hline$-.5189459 \times 10^{-14}$ & $-.3039762 \times 10^{-13}$ & $-.1909882 \times 10^{-13}$ \\
\hline
\end{tabular}


Table 3: Correctors Not Energized - Monitors No. 40 - 80 Shown.

\begin{tabular}{|c|c|c|c|}
\hline $\begin{array}{c}\text { Monitor } \\
\text { No. }\end{array}$ & $\begin{array}{c}\text { Uncorrected Orbit } \\
\text { No Apert. Restr. } \\
Y_{u}(\mathrm{~mm})\end{array}$ & $\begin{array}{c}\text { Kick Angle } \\
\theta(\mathrm{mrad})\end{array}$ & $\begin{array}{c}\text { Uncorrected Orbit } \\
\text { Apert. Restr. } \\
Y_{u}(\mathrm{~mm})\end{array}$ \\
\hline 40 & 7.73657 & 0.00000 & 7.73657 \\
\hline 41 & 19.24344 & 0.00000 & 19.24344 \\
\hline 42 & -4.67916 & 0.00000 & -4.67916 \\
\hline 43 & -20.23548 & 0.00000 & -20.23548 \\
\hline 44 & 0.15661 & 0.00000 & 0.15661 \\
\hline 45 & 21.20966 & 0.00000 & 21.20966 \\
\hline 46 & -0.58554 & 0.00000 & -0.58554 \\
\hline 47 & -19.99434 & 0.00000 & -19.99434 \\
\hline 48 & 2.19936 & 0.00000 & 2.19936 \\
\hline 49 & 19.62554 & 0.00000 & 19.62554 \\
\hline 50 & 0.06032 & 0.00000 & 0.06032 \\
\hline 51 & -53.64707 & 0.00000 & -53.64707 \\
\hline 52 & -37.62993 & 0.00000 & \\
\hline 53 & 44.59404 & 0.00000 & \\
\hline 54 & -0.56658 & 0.00000 & \\
\hline 55 & -13.38384 & 0.00000 & \\
\hline 56 & -32.71763 & 0.00000 & \\
\hline 57 & -2.43772 & 0.00000 & \\
\hline 58 & 28.18619 & 0.00000 & \\
\hline 59 & 2.82418 & 0.00000 & \\
\hline 60 & -26.72156 & 0.00000 & \\
\hline 61 & -8.63079 & 0.00000 & \\
\hline 62 & 26.46661 & 0.00000 & \\
\hline 63 & 10.55636 & 0.00000 & \\
\hline 64 & -26.16312 & 0.00000 & \\
\hline 65 & -16.48604 & 0.00000 & \\
\hline 66 & 30.68005 & 0.00000 & \\
\hline 67 & 12.46257 & 0.00000 & \\
\hline 68 & -26.83458 & 0.00000 & \\
\hline 69 & -15.15737 & 0.00000 & \\
\hline 70 & 7.67792 & 0.00000 & \\
\hline 71 & 18.75494 & 0.00000 & \\
\hline 72 & -31.72159 & 0.00000 & \\
\hline 73 & 11.04590 & 0.00000 & \\
\hline 74 & 20.11037 & 0.00000 & \\
\hline 75 & 18.74812 & 0.00000 & \\
\hline 76 & 19.68837 & 0.00000 & \\
\hline 77 & -23.26267 & 0.00000 & \\
\hline 78 & -25.20704 & 0.00000 & \\
\hline 79 & 22.98045 & 0.00000 & \\
\hline 80 & 21.11573 & 0.00000 & \\
\hline
\end{tabular}


Table 4: Some Correctors Energized - Monitors No. 40 - 80 Shown.

\begin{tabular}{|c|c|c|c|}
\hline $\begin{array}{c}\text { Monitor } \\
\text { No. }\end{array}$ & $\begin{array}{c}\text { Uncorrected Orbit } \\
\text { Apert. Restr. } \\
Y_{u}(\mathrm{~mm})\end{array}$ & $\begin{array}{c}\text { Kick Angle } \\
\theta(\mathrm{mrad})\end{array}$ & $\begin{array}{c}\text { First Correction } \\
\text { Apert. Restr. } \\
Y_{c}(\mathrm{~mm})\end{array}$ \\
\hline 40 & 7.73657 & -0.01600 & -0.06499 \\
\hline 41 & 19.24344 & -0.04459 & -0.39094 \\
\hline 42 & -4.67916 & 0.01708 & 0.55022 \\
\hline 43 & -20.23548 & 0.07383 & 0.15293 \\
\hline 44 & 0.15661 & -0.02162 & -0.07505 \\
\hline 45 & 21.20966 & 0.02924 & -0.29180 \\
\hline 46 & -0.58554 & -0.02627 & 0.61283 \\
\hline 47 & -19.99434 & -0.05143 & 0.13973 \\
\hline 48 & 2.19936 & -0.01457 & 0.05236 \\
\hline 49 & 19.62554 & -0.00463 & -0.23980 \\
\hline 50 & 0.06032 & 0.03724 & -0.05380 \\
\hline 51 & -53.64707 & 0.00000 & 0.43460 \\
\hline 52 & & 0.00000 & 0.39067 \\
\hline 53 & $:$ & -0.08708 & -1.95595 \\
\hline 54 & & 0.00000 & -4.79465 \\
\hline 55 & & 0.00000 & 0.27276 \\
\hline 56 & & 0.00000 & 7.39266 \\
\hline 57 & & 0.00000 & 7.70180 \\
\hline 58 & $!$ & 0.00000 & -6.67121 \\
\hline 59 & & 0.00000 & -5.93445 \\
\hline 60 & & 0.00000 & 6.11429 \\
\hline 61 & . & 0.00000 & 5.66983 \\
\hline 62 & $:$ & 0.00000 & -7.92776 \\
\hline 63 & & 0.00000 & -1.71124 \\
\hline 64 & & 0.00000 & 5.29259 \\
\hline 65 & & 0.00000 & 1.71385 \\
\hline 66 & & 0.00000 & -2.36258 \\
\hline 67 & & 0.00000 & -3.42087 \\
\hline 68 & & 0.00000 & 2.79056 \\
\hline 69 & & 0.00000 & 5.87633 \\
\hline 70 & & 0.00000 & 0.58306 \\
\hline 71 & & 0.00000 & -2.63172 \\
\hline 72 & & 0.00000 & \\
\hline 73 & & 0.00000 & \\
\hline 74 & & 0.00000 & \\
\hline 75 & & 0.00000 & \\
\hline 76 & & 0.00000 & \\
\hline 77 & & 0.00000 & \\
\hline 78 & & 0.00000 & \\
\hline 79 & & 0.00000 & \\
\hline 80 & & 0.00000 & \\
\hline
\end{tabular}




\section{THIRD INJECTION ATTEMPT}

Table 5: Full Turn Around Achieved with Some Correctors Energized (70 out of 123) - Monitors No. 40-80 Shown.

\begin{tabular}{|c|c|c|c|}
\hline $\begin{array}{l}\text { Monitor } \\
\text { No. }\end{array}$ & $\begin{array}{c}\text { Previous Correction } \\
Y_{c}(\mathrm{~mm}) \\
\end{array}$ & $\begin{array}{c}\text { Kick Angle } \\
\theta(\mathrm{mrad})\end{array}$ & $\begin{array}{c}\text { Present Correction } \\
Y_{c}(\mathrm{~mm}) \\
\end{array}$ \\
\hline 40 & -0.06499 & -0.01406 & 0.00536 \\
\hline 41 & -0.39094 & -0.05500 & 0.00085 \\
\hline 42 & 0.55022 & 0.02274 & -0.00552 \\
\hline 43 & 0.15293 & 0.06405 & -0.00199 \\
\hline 44 & -0.07505 & -0.01860 & 0.00641 \\
\hline 45 & -0.29180 & 0.01781 & 0.00210 \\
\hline 46 & 0.61283 & -0.02230 & -0.00699 \\
\hline 47 & 0.13973 & -0.06510 & -0.00576 \\
\hline 48 & 0.05236 & -0.01159 & 0.00686 \\
\hline 49 & -0.23980 & -0.00468 & 0.00764 \\
\hline 50 & -0.05380 & 0.03724 & -0.00420 \\
\hline 51 & 0.43460 & -0.00575 & -0.03458 \\
\hline 52 & 0.39067 & 0.02851 & -0.02355 \\
\hline 53 & -1.95595 & 0.00217 & 0.03336 \\
\hline 54 & -4.79465 & 0.03987 & -0.00549 \\
\hline 55 & $\quad 0.27276$ & 0.05547 & -0.00871 \\
\hline 56 & 7.39266 & -0.03455 & -0.01092 \\
\hline 57 & $\quad 7.70180$ & -0.00207 & -0.10412 \\
\hline 58 & -6.67121 & -0.04424 & 1.00845 \\
\hline 59 & -5.93445 & 0.00451 & -0.30856 \\
\hline 60 & $\quad 6.11429$ & 0.01137 & -0.20256 \\
\hline 61 & $\quad 5.66983$ & 0.04607 & -0.24598 \\
\hline 62 & -7.92776 & -0.09209 & 0.97850 \\
\hline 63 & -1.71124 & 0.05239 & -0.32273 \\
\hline 64 & $\quad 5.29259$ & 0.00733 & -0.73500 \\
\hline 65 & $\quad 1.71385$ & -0.05941 & -0.20721 \\
\hline 66 & -2.36258 & 0.03289 & 0.93067 \\
\hline 67 & -3.42087 & -0.01196 & 0.05824 \\
\hline 68 & $\quad 2.79056$ & -0.03419 & -0.50199 \\
\hline 69 & $: \quad 5.87633$ & 0.01818 & -0.37403 \\
\hline 70 & 0.58306 & 0.00388 & 0.11162 \\
\hline 71 & -2.63172 & 0.00000 & 0.38828 \\
\hline 72 & & 0.00000 & 0.24415 \\
\hline 73 & & 0.00000 & -1.08713 \\
\hline 74 & & 0.00000 & -0.91391 \\
\hline 75 & & 0.00000 & 2.67388 \\
\hline 76 & & 0.00000 & 4.87007 \\
\hline 77 & & 0.00000 & -1.03826 \\
\hline 78 & & 0.00000 & -5.24478 \\
\hline 79 & ; & 0.00000 & 0.58502 \\
\hline 80 & & 0.00000 & 3.54740 \\
\hline
\end{tabular}


Table 6: Full Turn Around Achieved with Some Correctors Energized (70 out of 123) - Monitors No. 1 - 40 Shown.

\begin{tabular}{|c|c|c|c|}
\hline $\begin{array}{l}\text { Monitor } \\
\text { No. }\end{array}$ & $\begin{array}{c}\text { Previous Correction } \\
Y_{c}(\mathrm{~mm}) \\
\end{array}$ & $\begin{array}{c}\text { Kick Angle } \\
\theta(\mathrm{mrad})\end{array}$ & $\begin{array}{c}\text { Present Correction } \\
Y_{c}(\mathrm{~mm}) \\
\end{array}$ \\
\hline 1 & 0.00000 & -0.01037 & 0.00000 \\
\hline 2 & 0.00012 & 0.01939 & 0.00000 \\
\hline 3 & -0.00322 & 0.10082 & 0.00000 \\
\hline 4 & 0.00461 & 0.05095 & -0.00002 \\
\hline 5 & 0.06468 & -0.06554 & 0.00006 \\
\hline 6 & 0.03244 & -0.01518 & -0.00021 \\
\hline 7 & 0.00106 & 0.03079 & -0.00005 \\
\hline 8 & 0.04645 & -0.02512 & 0.00023 \\
\hline 9 & 0.00265 & -0.05545 & 0.00005 \\
\hline 10 & -0.11144 & -0.01178 & -0.00043 \\
\hline 11 & -0.07876 & -0.08334 & -0.00031 \\
\hline 12 & 0.09353 & 0.03476 & 0.00032 \\
\hline 13 & -0.00087 & 0.03690 & 0.00004 \\
\hline 14 & -0.02626 & 0.01686 & -0.00010 \\
\hline 15 & -0.06100 & 0.01591 & -0.00030 \\
\hline 16 & -0.15089 & -0.02839 & -0.00009 \\
\hline 17 & 0.40319 & 0.10286 & 0.00055 \\
\hline 18 & 0.09845 & 0.00991 & 0.00311 \\
\hline 19 & 0.04078 & 0.08059 & -0.00041 \\
\hline 20 & -0.11795 & 0.02956 & -0.00357 \\
\hline 21 & -0.02502 & 0.03713 & 0.00020 \\
\hline 22 & 0.33826 & -0.09700 & 0.00234 \\
\hline 23 & 0.12625 & 0.03181 & 0.00562 \\
\hline 24 & -0.01145 & -0.09538 & -0.00020 \\
\hline 25 & 0.14595 & 0.01230 & -0.00588 \\
\hline 26 & 0.01875 & 0.08449 & 0.00004 \\
\hline 27 & -0.09835 & 0.05948 & 0.00527 \\
\hline 28 & -0.07398 & 0.04069 & -0.00301 \\
\hline 29 & 0.02153 & 0.05545 & -0.00456 \\
\hline 30 & 0.07069 & 0.01683 & -0.00431 \\
\hline 31 & -0.09619 & 0.01415 & 0.01735 \\
\hline 32 & 0.02281 & -0.07835 & -0.01161 \\
\hline 33 & 0.04832 & 0.02094 & -0.01689 \\
\hline 34 & 0.06180 & -0.01386 & -0.00380 \\
\hline 35 & 0.07078 & -0.04864 & 0.00156 \\
\hline 36 & -0.08465 & -0.00787 & 0.00554 \\
\hline 37 & -0.21867 & 0.02373 & 0.00014 \\
\hline 38 & 0.44014 & -0.01824 & -0.00524 \\
\hline 39 & 0.30171 & 0.01497 & -0.00160 \\
\hline 40 & -0.06499 & -0.01406 & 0.00536 \\
\hline
\end{tabular}


Table 7: All Correctors Energized - Betatron Motion Develops - Monitors No. 1 - 40 Shown.

\begin{tabular}{|c|c|c|c|}
\hline $\begin{array}{c}\text { Monitor } \\
\text { No. }\end{array}$ & $\begin{array}{c}\text { Previous Correction } \\
Y_{c}(\mathrm{~mm})\end{array}$ & $\begin{array}{c}\text { Kick Angle } \\
\theta(\mathrm{mrad})\end{array}$ & $\begin{array}{c}\text { Present Correction } \\
Y_{c}(\mathrm{~mm})\end{array}$ \\
\hline 1 & 0.00000 & 0.00107 & 0.90711 \\
2 & 0.00000 & 0.01921 & -0.54440 \\
3 & -0.38473 & 0.10070 & -0.93042 \\
4 & -0.01508 & 0.05106 & 0.49901 \\
5 & 0.38400 & -0.06565 & 0.95543 \\
6 & 0.03497 & -0.01514 & -0.45898 \\
7 & -0.38249 & 0.03073 & -0.97617 \\
8 & -0.10198 & -0.02512 & 0.24068 \\
9 & 0.25785 & -0.05545 & 0.69459 \\
10 & $: 16976$ & -0.01178 & 1.81133 \\
11 & 0.77608 & -0.08334 & 1.14946 \\
12 & -1.22815 & 0.03476 & -2.15649 \\
13 & 0.30588 & 0.03690 & 0.78046 \\
14 & 0.30755 & 0.01686 & 0.50926 \\
15 & 0.18458 & 0.01590 & -0.18529 \\
16 & -0.37262 & -0.02840 & -1.02370 \\
17 & -0.10378 & 0.10282 & 0.26571 \\
18 & 0.36700 & 0.00989 & 1.03913 \\
19 & 0.12448 & 0.08062 & -0.21116 \\
20 & -0.36014 & 0.02960 & -1.05002 \\
21 & -0.14559 & 0.03718 & 0.14778 \\
22 & 0.35241 & -0.09712 & 1.05956 \\
23 & 0.16494 & 0.03180 & -0.09329 \\
24 & -0.34268 & -0.09540 & -1.06311 \\
25 & -0.18318 & 0.01232 & 0.03191 \\
26 & 0.33316 & 0.08445 & 1.06679 \\
27 & 0.20259 & 0.05955 & 0.02450 \\
28 & -0.43832 & 0.04069 & -1.32074 \\
29 & -0.33279 & 0.05545 & -0.65928 \\
30 & -0.18988 & 0.01683 & -0.11797 \\
31 & 1.23001 & 0.01415 & 2.36322 \\
32 & -0.92611 & -0.07835 & -1.99867 \\
33 & -1.31006 & 0.02094 & -2.75633 \\
34 & -0.16834 & -0.01386 & -0.10843 \\
35 & 0.30764 & -0.04864 & 1.01032 \\
36 & 0.25953 & -0.00789 & 0.21416 \\
37 & -0.27897 & 0.02376 & -1.03889 \\
38 & -0.27216 & -0.01822 & -0.26849 \\
39 & 0.26811 & 0.01498 & 1.02886 \\
40 & 0.28415 & -0.01404 & 0.31276 \\
\hline & & & \\
\hline
\end{tabular}


Table 8: Several Successive Adjustments of Orbit Correctors Implemented. Initial Injection Conditions Also Adjusted. Betatron Motion Magnitude Drops. Monitors No. $1-40$ Shown.

\begin{tabular}{|c|c|c|c|}
\hline $\begin{array}{l}\text { Monitor } \\
\text { No. }\end{array}$ & $\begin{array}{c}\text { Previous Correction } \\
Y_{c}(\mathrm{~mm}) \\
\end{array}$ & $\begin{array}{c}\text { Kick Angle } \\
\theta(\mathrm{mrad})\end{array}$ & $\begin{array}{c}\text { Present Correction } \\
Y_{c}(\mathrm{~mm}) \\
\end{array}$ \\
\hline 1 & 0.00000 & 0.00103 & -0.00190 \\
\hline 2 & 0.55255 & 0.01912 & -0.00256 \\
\hline 3 & 0.02646 & 0.10084 & 0.00171 \\
\hline 4 & -0.55097 & 0.05096 & 0.00319 \\
\hline 5 & -0.04966 & -0.06552 & -0.00129 \\
\hline 6 & 0.54890 & -0.01519 & -0.00249 \\
\hline 7 & 0.07506 & 0.03080 & 0.00058 \\
\hline 8 & -0.49408 & -0.02512 & 0.00250 \\
\hline 9 & -0.08808 & -0.05545 & -0.00022 \\
\hline 10 & 0.97531 & -0.01178 & -0.00726 \\
\hline 11 & 0.70072 & -0.08334 & -0.00506 \\
\hline 12 & -0.76258 & 0.03476 & 0.00643 \\
\hline 13 & -0.05984 & 0.03690 & -0.00046 \\
\hline 14 & 0.22253 & 0.01686 & -0.00175 \\
\hline 15 & 0.63737 & 0.01591 & -0.00335 \\
\hline 16 & 0.14860 & -0.02842 & 0.00039 \\
\hline 17 & -0.52813 & 0.10280 & 0.00130 \\
\hline 18 & $:-0.17747$ & 0.00990 & -0.00019 \\
\hline 19 & 0.51772 & 0.08060 & -0.00171 \\
\hline 20 & 0.20611 & 0.02952 & 0.00036 \\
\hline 21 & -0.50781 & 0.03715 & -0.00028 \\
\hline 22 & -0.23368 & -0.09713 & -0.00060 \\
\hline 23 & 0.49413 & 0.03176 & -0.00079 \\
\hline 24 & 0.26171 & -0.09538 & -0.00008 \\
\hline 25 & -0.48028 & 0.01229 & 0.00068 \\
\hline 26 & -0.28756 & 0.08451 & -0.00033 \\
\hline 27 & 0.46413 & 0.05950 & -0.00022 \\
\hline 28 & 0.29414 & 0.04069 & -0.00079 \\
\hline 29 & -0.12843 & 0.05545 & -0.00026 \\
\hline 30 & -0.33771 & 0.01683 & 0.00011 \\
\hline 31 & 0.54998 & 0.01415 & 0.00089 \\
\hline 32 & -0.18947 & -0.07835 & -0.00089 \\
\hline 33 & -0.34069 & 0.02094 & -0.00118 \\
\hline 34 & -0.29547 & -0.01386 & 0.00009 \\
\hline 35 & -0.29182 & -0.04864 & 0.00064 \\
\hline 36 & 0.40739 & -0.00788 & -0.00010 \\
\hline 37 & 0.39047 & 0.02371 & -0.00062 \\
\hline 38 & -0.38494 & -0.01822 & -0.00121 \\
\hline 39 & -0.40708 & 0.01496 & 0.00013 \\
\hline 40 & 0.36612 & -0.01404 & 0.00091 \\
\hline
\end{tabular}


Figure 1

Phase Space Plot at Physical Q90 Exit

Horizontal Plane. No. of Turns: 50

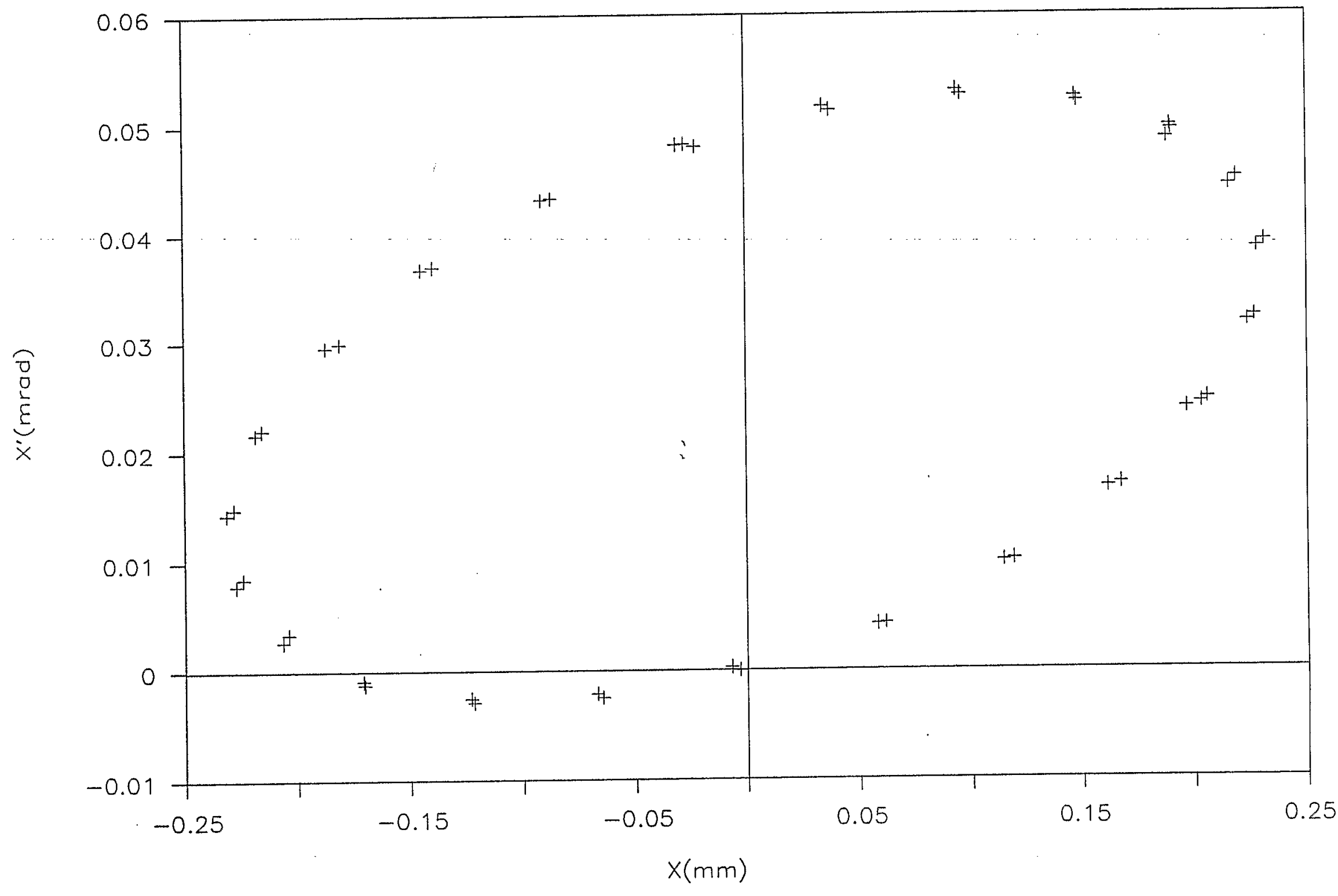

